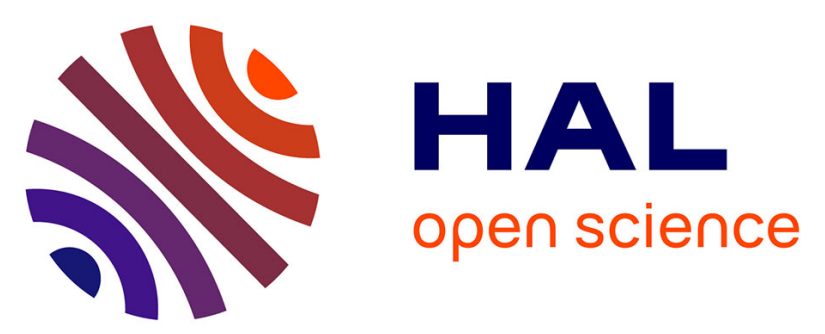

\title{
Accurate narrowband LPWA ranging: Principles, performance in AWGN and multipath channels
}

Florian Wolf, Sébastien de Rivaz, François Dehmas, Valérian Mannoni, Vincent Berg, Jean-Pierre Cances

\section{- To cite this version:}

Florian Wolf, Sébastien de Rivaz, François Dehmas, Valérian Mannoni, Vincent Berg, et al.. Accurate narrowband LPWA ranging: Principles, performance in AWGN and multipath channels. EuCNC 2020 - 29th European Conference on Networks and Communications, Jun 2020, Durbovnick, Croatia. pp.148-153, 10.1109/EuCNC48522.2020.9200944 . cea-03199084

\section{HAL Id: cea-03199084 https://hal-cea.archives-ouvertes.fr/cea-03199084}

Submitted on 15 Apr 2021

HAL is a multi-disciplinary open access archive for the deposit and dissemination of scientific research documents, whether they are published or not. The documents may come from teaching and research institutions in France or abroad, or from public or private research centers.
L'archive ouverte pluridisciplinaire HAL, est destinée au dépôt et à la diffusion de documents scientifiques de niveau recherche, publiés ou non, émanant des établissements d'enseignement et de recherche français ou étrangers, des laboratoires publics ou privés. 


\title{
Accurate Narrowband LPWA Ranging: Principles, Performance in AWGN and Multipath Channels
}

\author{
Florian Wolf*\|, Sébastien de Rivaz*, François Dehmas*, \\ Valérian Mannoni*, Vincent Berg* and Jean-Pierre Cances" \\ * CEA-Leti Minatec Campus, 17 rue des Martyrs, 38054 Grenoble Cedex 09, France \\ Email: \{florian.wolf, sebastien.derivaz, francois.dehmas, valerian.mannoni, vincent.berg\}@cea.fr \\ \| Université de Limoges, CNRS, XLIM, UMR 7252, 87000 Limoges, France \\ Email: \{florian.wolf, jean-pierre.cances\}@xlim.fr
}

\begin{abstract}
Today approaches of radio localization for Low Power Wide Area networks do not provide sufficiently accurate ranging required by applications such as wearable health monitoring. Coherent multi-channel ranging or Phase-of-Flight (PoF) ranging provides significantly improved temporal resolution through the aggregation of sequentially transmitted narrowband signals for the same level coverage performance as legacy Time-of-Flight (ToF) ranging. This paper compares the performance of $\mathrm{PoF}$ and $\mathrm{ToF}$ under additive white Gaussian channels with both simulations and laboratory measurements. Field trials have been performed in a multipath outdoor environment and strong ranging biases are observed. Ranging bias estimators are introduced and evaluated to mitigate these damaging effects: thanks to the new approach, accuracy of $\mathbf{3 0} \mathrm{m}$ in $90 \%$ of the cases may be obtained. This compares to $250 \mathrm{~m}$ when legacy ToF is considered.

Index Terms-Narrowband localization, LPWA, range estimation, frequency hopping, multipath classification/mitigation
\end{abstract}

\section{INTRODUCTION}

Low Power Wide Area (LPWA) networks are emerging communication technologies enabling wireless connectivity on a large variety of objects in the context of the Internet of Things (IoT). Long-range communication of both, proprietary (e.g. LoRa, Sigfox) and standardized solutions (NB-IoT), is achieved thanks to low levels of receiver sensitivity obtained by low data rates and narrowband modulation schemes.

One promising usage of LPWA networks is envisaged for wearable health monitoring devices to allow remote patient diagnostics and enable applications such as emergency alerts in case of e.g. heart strokes. LPWA radios are suited for these applications due to their small size, low-power and low-cost, while providing indoor and outdoor coverage.

Localization of the patient is essential to facilitate the work of emergency services. GNSS based solutions should be excluded because of extra power consumption and unavailability for indoor operation. A power efficient approach could derive position information from the same radio signal as used to transmit health-monitoring data. However, current approaches are not accurate enough to meet requirements of the application in indoor or outdoor environments [1].

Fingerprinting methods based on Received Signal Strength Indicator (RSSI) can achieve positioning errors smaller than $500 \mathrm{~m}$ when applied to a metropolitan LPWA network [2]. Although straightforward to implement, this approach requires large up-to-date fingerprint databases. Alternatively, Time-of-Arrival (ToA) based localization is bound by the signal bandwidth used to estimate ranging information [3]. Precise LPWA localization is challenging due to narrowband modulation schemes and the difficulty to resolve multipath. Time-Difference-of-Arrival (TDoA), which is more suited to LPWA networks as it relaxes synchronization constraints, achieves $250 \mathrm{~m}$ accuracy in this context [4] and could be further improved with new chipsets [5] that rely on increased instantaneous bandwidth at the tradeoff of coverage.

Coherent multi-channel ranging also called Phase-of-Flight (PoF) sequentially aggregates multiple transmitted narrowband signals on different frequencies and virtually increases signal bandwidth [3] to improve ranging resolution. This technique maintains coverage properties and is adapted to narrowband LPWA signals. Various implementations of PoF [6]-[8] have demonstrated a higher accuracy over other time based ranging techniques. However, these studies have focused on short-range technologies (i.e. WiFi, Zigbee, RFID) and have mostly been applied to indoor propagation scenarios.

Besides, localization is highly degraded in the presence of multipath propagation as ToA metrics are altered by signal reflections and blockages. In this case, reliability information can be provided to discard or mitigate the resulting biased estimates. This issue is solved in the context of ultra-wideband radios by extracting the channel impulse response (CIR) to classify Non-Line of Sight (NLoS) measurements [9]. The derived reliability information can be fed to the location solver to weight ranging estimates. Such methods do not apply to narrowband LPWA signals since the resolution of the CIR is insufficient but should be considered for PoF.

This work demonstrates how precise PoF ranging applied to narrowband LPWA signals and combined with range estimation reliability metrics enables accurate localization.

The contributions of this paper are thus threefold:

- Characterization of the precision gain of PoF over ToF techniques for LPWA narrowband signals.

- Combination of multi-channel ranging and CIR based methods to extract reliability information and eliminate 
inaccurate estimates from the position estimation process.

- Field trials with a transceiver [10] demonstrating how a CIR and delay spread based multipath/NLoS classification technique can mitigate ranging biases.

This paper is organized as follows. Section II introduces PoF ranging with a reliability metric. A LPWA ranging transceiver testbed is presented in Section III and its performance under Additive White Gaussian Noise (AWGN) in Section IV. Multipath field trial experimentations and results are presented in Section V. Section VI concludes the paper and outlines perspectives.

\section{Coherent Multi-Channel Ranging}

\section{A. Principles of Phase-of-Flight (PoF)}

A time-frequency representation of the multi-channel ranging signaling process [10] is given in Fig. 1 where two nodes sequentially perform a two-way packet exchange on different frequencies $f_{c}=f_{\mathrm{R}}+c \Delta f, c \in[0, C-1]$. With constant carrier frequency $f_{\mathrm{R}}$, a channel spacing $\Delta f$ and a total number of $C$ channels. The two-way exchange is designed to synchronize both nodes that do not share a common reference clock. Packets of instantaneous narrow bandwidth $B W_{\text {sym }}$ and characterized by transmission times $T_{\mathrm{p}}$ provide long-range connectivity.

The cross correlation of the received signal $r_{c}^{[X]}$ with the transmitted known preamble $s_{0}$ for both nodes $X \in\{1,2\}$ is given by

$$
\begin{gathered}
\Omega_{r_{c}, s_{0}}^{[X]}\left[t_{\mathrm{A}}, \delta_{\mathrm{f}}\right]=\sum_{k=0}^{K_{\mathrm{S}}-1}\left(r_{c}^{[X]}\left[k-\frac{t_{\mathrm{A}}}{T_{\mathrm{S}}}\right]\right)^{*} s_{0}[k] \\
\cdot e^{-j 2 \pi \delta_{\mathrm{f}} f_{c} k T_{\mathrm{S}}},
\end{gathered}
$$

with complex conjugate $(\cdot)^{*}$, sampling interval $T_{\mathrm{S}}$ over $K_{\mathrm{S}}$ samples. For each channel $c$, estimates of the relative Carrier Frequency Offset $(\mathrm{CFO}) \delta_{\mathrm{f}}$, the ToA $t_{\mathrm{A}}$, the

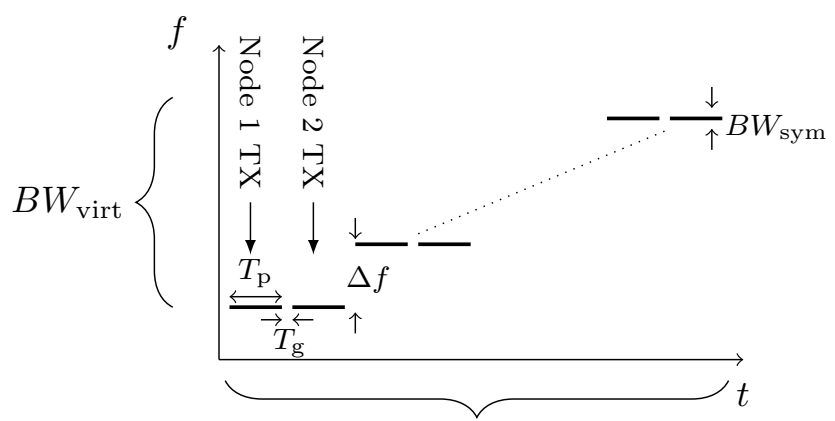

$C$ two-way amplitude/phase channel estimates

Fig. 1. Multi-channel ranging signaling process for $C$ sequentially aggregated narrowband $\left(B W_{\text {sym }}\right)$ channels form a virtual bandwidth $B W_{\text {virt }}=(C-1) \Delta f$.
Phase-of-Arrival (PoA) $\phi_{c}^{[X]}$, and the amplitude $A_{c}^{[X]}$ are given, according to [7], by

$$
\begin{aligned}
& {\widehat{\delta_{\mathrm{f}}}}^{[X]}=\underset{\delta_{\mathrm{f}}}{\arg \max } \operatorname{tax}_{\mathrm{A}}\left|\Omega_{\mathrm{f}}^{[X]}\left[t_{r_{c}, s_{0}}, \delta_{\mathrm{f}}\right]\right|, \\
& {\widehat{t_{\mathrm{A} c}}}^{[X]}=\underset{t_{\mathrm{A}}}{\arg \max }\left|\Omega_{t_{\mathrm{A}}, \delta_{\mathrm{f}}}^{[X]}\left[t_{r_{c}, s_{0}}, \delta_{\mathrm{f}}\right]\right|, \\
& {\widehat{\phi_{c}}}^{[X]}=\angle\left\{\Omega_{r_{c}, s_{0}}^{[X]}\left[{\widehat{t_{\mathrm{A}}}}^{[X]},{\widehat{\delta_{\mathrm{f}}}}^{[X]}\right]\right\}, \\
& {\widehat{A_{c}}}^{[X]}=\left|\Omega_{r_{c}, s_{0}}^{[X]}\left[\widehat{t_{\mathrm{A}}}[X],{\widehat{\delta_{\mathrm{f}}}}^{[X]}\right]\right| .
\end{aligned}
$$

The PoA estimates for the received signal from node 1 (resp. 2) at node 2 (resp. 1) are given by

$$
\begin{aligned}
\phi_{c}^{\left[R_{2}\right]}= & \phi_{\mathrm{R}, c}^{\left[T_{1}\right]}-2 \pi f_{c}\left(\tau_{0}+t_{0}\right)+\varphi_{c}-\phi_{\mathrm{R}, c}^{\left[R_{2}\right]}, \\
\phi_{c}^{\left[R_{1}\right]}= & \phi_{\mathrm{R}, c}^{\left[T_{2}\right]}-2 \pi f_{c}\left(\tau_{0}-t_{0}\right)+\varphi_{c}-\phi_{\mathrm{R}, c}^{\left[R_{1}\right]} \\
& +2 \pi f_{c} \delta_{\mathrm{f}}\left(T_{\mathrm{p}}+T_{\mathrm{g}}+\epsilon_{t}\right),
\end{aligned}
$$

with propagation delay $\tau_{0}$, radio channel phase $\varphi_{c}$ and initial transceiver oscillator phase $\phi_{\mathrm{R}, c^{[}}^{[X]}$. Furthermore, the return packet in ( $3 b)$ integrates a phase error due to the $\mathrm{CFO} \delta_{\mathrm{f}}$ and because the phase measurement is taken $T_{\mathrm{p}}+T_{\mathrm{g}}+\epsilon_{t}$ after PoA $\phi_{c}^{\left[R_{2}\right]}$. This phase error can be corrected with a CFO estimation and the a priori knowledge of $T_{\mathrm{p}}+T_{\mathrm{g}}$. CFO estimation precision should fulfill $\epsilon_{\delta_{\mathrm{f}}}<\epsilon_{\tau_{0}} /\left(T_{\mathrm{p}}+T_{\mathrm{g}}\right)$, when maximum acceptable range error is $\epsilon_{\tau_{0}}$. The time synchronization error $\epsilon_{t}$ may be considered negligible as $\delta_{\mathrm{f}} \epsilon_{t} \ll \tau_{0}$. The time offset $t_{0}$ between both nodes is cancelled when combining (3a) and (3b) as Phase-of-Flight (PoF)

$$
\phi_{c}^{[1,2]}=\phi_{c}^{\left[R_{2}\right]}+\phi_{c}^{\left[R_{1}\right]}=-4 \pi f_{c} \tau_{0}+2 \varphi_{c}+\Delta \phi_{\mathrm{R}, c},
$$

with $\Delta \phi_{\mathrm{R}, c}=\phi_{\mathrm{R}, c}^{\left[T_{1}\right]}-\phi_{\mathrm{R}, c}^{\left[R_{2}\right]}+\phi_{\mathrm{R}, c}^{\left[T_{2}\right]}-\phi_{\mathrm{R}, c}^{\left[R_{1}\right]}$. PoF imposes a transceiver architecture that ensures $\Delta \phi_{\mathrm{R}, c}=$ const. for all $c$.

For multipath propagation, the channel transfer function $^{1} H(f)$ can be reconstructed with amplitude and PoF estimates on channels $c \in[0, C-1]$ as follows

$$
H_{c}=\left.H(f)\right|_{\left(f=f_{\mathrm{R}}+c \Delta f\right)}={\widehat{A_{c}}}^{\left[R_{1}\right]}{\widehat{A_{c}}}^{\left[R_{2}\right]} e^{j{\widehat{\phi_{c}}}^{[1,2]}} .
$$

Applying the inverse discrete Fourier transform (iDFT) to $\boldsymbol{H}=\left[H_{0}, \ldots, H_{C-1}\right]$ results in a sampled version of the CIR $h(\tau)$ [7].

The theoretical range resolution for this multi-channel processing scheme is determined by the virtual bandwidth $B W_{\text {virt }}$ and equal to $\Delta R=c_{0} /(2(C-1) \Delta f)=c_{0} /\left(2 B W_{\text {virt }}\right) \quad$ [3] where $c_{0}$ is the speed of light.

\section{B. Range estimators and bias classification}

Two range estimators are thus compared for performance: PoF based on first path detection and ToF.

\footnotetext{
${ }^{1}$ The square of the channel transfer function $H^{2}(f)$ is measured due to two-way ranging.
} 
1) Legacy Time-of-Flight (ToF): Single channel ToF measurements $d_{\text {ToF,c }}$ may be obtained on each channel $c$. For a fair comparison to PoF based ranging, the median of $C$ single channel ToF measurements is given by $d_{\text {ToF,MC }}$. Calculating the median allows reducing the impact of outliers.

2) Phase-of-Flight - First path detection: The large and scalable virtual bandwidth $B W_{\text {virt }}$, compared to the instantaneous narrowband signal bandwidth $B W_{\text {sym }}$, allows resolving different propagation paths in the CIR to some extent. A first path detection algorithm accounts for scenarios when the direct path, corresponding to the true range, is not the strongest path. The range estimate $d_{\mathrm{PoF} \text {,first }}$ is given by searching for the first path above a certain threshold $\gamma_{\text {first }}$ relative to and in a certain range $R_{\text {first }}$ before the global maximum in the estimated channel impulse response $h(\tau)$.

For both techniques, temporal smoothing with a moving median over three measurements eliminates sporadic outliers on $d_{\mathrm{PoF}, \text { first }}$ and $d_{\mathrm{ToF}, \mathrm{MC}}$.

3) NLoS classification: Multi-channel measurements provide an estimate of the CIR. Although not as well resolved as in ultra-wideband systems, the CIR can be used to calculate macroscopic features such as the delay spread

$$
\sigma_{\tau}=\sqrt{\overline{\tau^{2}}-\left(\overline{\tau^{1}}\right)^{2}}
$$

with first and second moment $\overline{\tau^{1}}$ and $\overline{\tau^{2}}$ of the amplitude CIR $|h(\tau)|$. The delay spread estimate can be used to derive a reliability metric, which allows classifying range measurements as biased or bias free [9], depending on whether the delay spread exceeds a threshold $\zeta_{\text {opt }}$. Classifying a range estimate as biased and hence as unreliable is a valuable information in the process of localization, where a location solver can profit from a reliability indicator to select the set of range estimates to different base stations that dispose the least bias, i.e. are the most reliable.

\section{LPWA RANGING TRANSCEIVER TESTBED}

An experimental validation is realized in a real propagation channel. The transceiver testbed [10] illustrated in Fig. 2 has been used. It is composed of a Software Defined Radio $^{2}$ (SDR), radio frequency components and a GNSS module $^{3}$ to obtain the range reference (ground truth). Digital intermediate frequency up-/down-mixing stages are implemented into the SDR to coherently process a $10 \mathrm{MHz}$ bandwidth by sequentially selecting $1 \mathrm{MHz}$ channels. This architecture ensures that $\Delta \phi_{\mathrm{R}, c}=$ const.for all $c$ in (4).

Two transceiver testbeds perform a multi-channel two-way ranging protocol according to Fig. 1. For this purpose, the nodes exchange a Binary Phase Shift Keying (BPSK) preamble of chip rate $R_{\mathrm{c}}=10 \mathrm{kchip} / \mathrm{s}$ ou $B W_{\mathrm{sym}}=10 \mathrm{kHz}$ following a Gold code of chip length $N_{\mathrm{c}}=256 \mathrm{chips}$, with $T_{\mathrm{g}}=3 \mathrm{~ms}$. Subsequently, a CFO estimation precision $\epsilon_{\delta_{\mathrm{f}}}<\left(1 \mathrm{~m} / c_{0}\right) /\left(N_{\mathrm{c}} / R_{\mathrm{c}}+T_{\mathrm{g}}\right) \approx 0.1 \mathrm{ppm} \quad$ (parts

\footnotetext{
${ }^{2}$ Analog Devices AD9361 $2 \times 2$ TRX radio front end and a Zynq-045 Xilinx System on chip FPGA with integrated dual Cortex-A9 ARM processor.

${ }^{3} \mathrm{uBlox}$ C94-M8P application board.
}

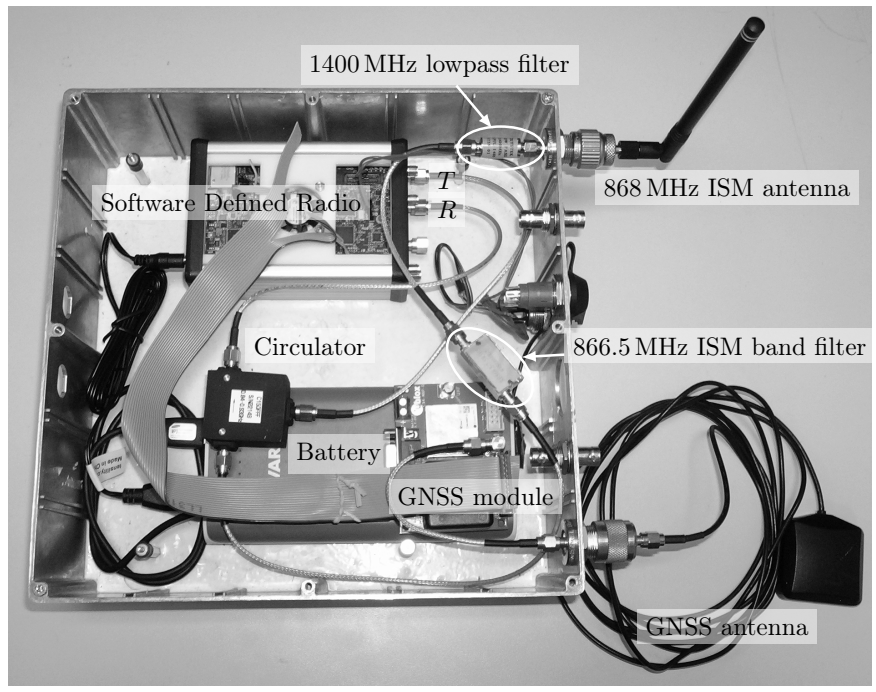

Fig. 2. Transceiver testbed composed of a SDR, radio frequency components, a GNSS module and a power supply.

per million) is required to achieve an error less than $1 \mathrm{~m}$. The modulation type and physical layer parameterization are similar to the Sigfox LPWA standard.

The two-way packet exchange is repeated $C=16$ times with a channel spacing $\Delta f=200 \mathrm{kHz}$ and $f_{\mathrm{R}}=866.212 \mathrm{MHz}$, resulting in a virtual bandwidth $B W_{\text {virt }}=3 \mathrm{MHz}$ and a range resolution $\Delta R=50 \mathrm{~m}$. Transmit power is set to $P_{\mathrm{TX}}=0 \mathrm{dBm}$. Radio frequency filters and the circulator, connecting $\mathrm{TX}$ and $\mathrm{RX}$ to the same antenna, add an attenuation $A_{\mathrm{RF}} \approx 5 \mathrm{~dB}$.

\section{PERformances in AN AWGN CHANNEL}

The signal model including $\mathrm{CFO}$ has been validated by numerical simulation and experimentation under Additive White Gaussian Noise (AWGN) channel conditions [10].

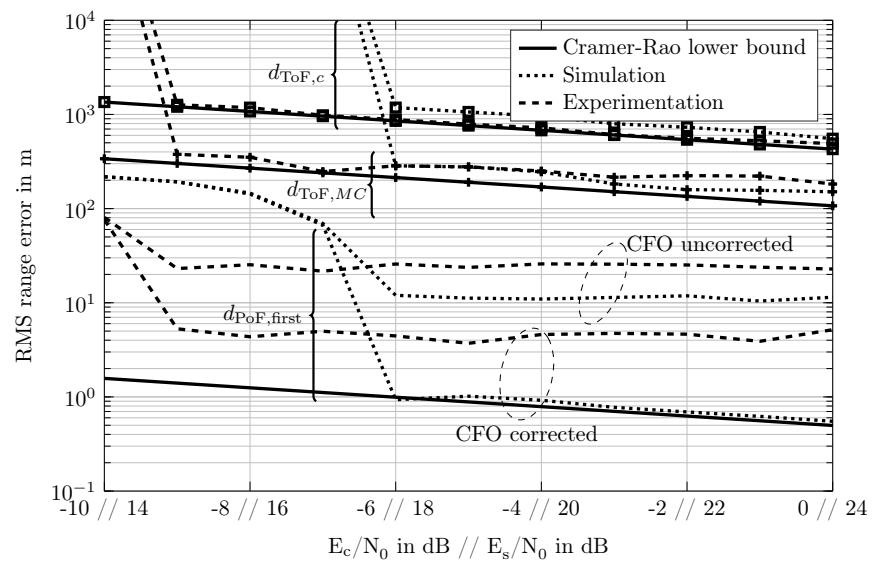

Fig. 3. Simulated and measured ranging error in a frequency flat channel with a signal bandwidth $B W_{\text {sym }}=10 \mathrm{kHz}, C=16$ channels, a virtual multi-channel bandwidth $B W_{\text {virt }}=3.0 \mathrm{MHz}$ and CFO. 


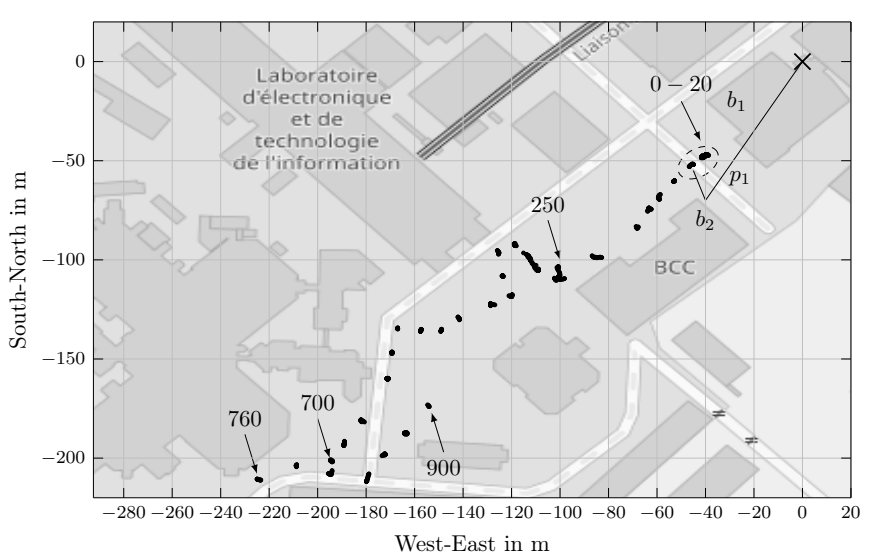

Fig. 4. GNSS ground truth positions $(\cdot)$ w.r.t. the roof testbed $(\times)$ at $(0,0)$.

Fig. 3 compares the Root-Mean-Square (RMS) ranging error over 100 realizations for both, simulated and through the testbed measured signals to Cramer-Rao Lower Bounds (CRLBs) as a function of the preamble symbol energy to noise spectral density ratio $E_{\mathrm{s}} / N_{0}$.

Simulation consider a random CFO $\left|\delta_{\mathrm{f}}\right| \leq 5 \mathrm{ppm}$ while experimentation is performed with a fixed experimentally measured CFO of $\delta_{\mathrm{f}} \approx 5 \mathrm{ppm}$. Calibration of implementation specific constant delays and phase shifts is performed for an $E_{\mathrm{s}} / N_{0}=20 \mathrm{~dB}$. In simulation, $\mathrm{CFO}$ can be corrected by appropriate compensation of the error terms in (3b), whereas experimentation with $\mathrm{CFO}$ correction levels out at a PoF ranging precision of $5 \mathrm{~m}$, due to jitter and time varying CFO. PoF measurements $d_{\mathrm{PoF}, \text { first }}$ clearly outperform ToF measurements $d_{\text {ToF,MC }}$, with respectively $5 \mathrm{~m}$ and $200 \mathrm{~m}$ error for sufficient $E_{\mathrm{s}} / N_{0}$. The cabled setup, recreates a LoS propagation channel and illustrates the gain of processing sequentially transmitted narrowband signals to increase bandwidth virtually.

Furthermore, the results reveal that precision converges ${ }^{4}$ for $E_{\mathrm{s}} / N_{0}>18 \mathrm{~dB}$ or $\left(E_{\mathrm{c}} / N_{0}\right)_{0}=-6 \mathrm{~dB}$. This operation point yields a typical LPWA link budget of $127.5 \mathrm{~dB}$ which is equivalent to a $65 \mathrm{~km}$ free-space coverage.

\section{FiELD TRIALS IN A MULTiPATH ENVIRONMENT}

Fig. 4 depicts the measurement positions in a quasi stationary scenario with transmission ranges of up to $R=$ $300 \mathrm{~m}$ between the base station $(\times)$, located on a $26 \mathrm{~m}$ high office building roof $b_{1}$ and the LPWA node $(\cdot)$ at positions 0 to 900 as indicated on the map. Measurements close to the building $b_{1}$ are in NLoS as the LoS to the base station on the roof is obstructed by the building itself. A total of 900 measurements has been performed.

Fig. 5a depicts ranging errors for the first path detection $\quad d_{\mathrm{PoF}, \text { first }}$ with parameters $\gamma_{\text {first }}=-7 \mathrm{~dB}$ and $R_{\text {first }}=300 \mathrm{~m}$, which are obtained through minimization of the ranging error in $90 \%$ of the cases. For comparison,

\footnotetext{
${ }^{4}$ The $3 \mathrm{~dB}$ mismatch between experimentation and simulation is due to measurement uncertainties (e.g. $P_{\mathrm{TX}}, A_{\mathrm{RF}}$ ).
}

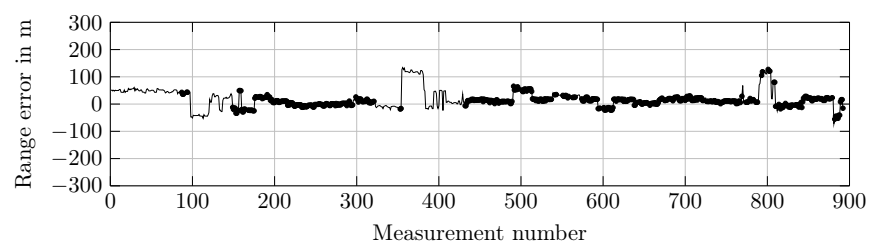

(a) PoF $d_{\mathrm{PoF} \text {,first }}$ ranging error with NLoS mitigation and classification $\sigma_{\tau}<\zeta_{\text {opt }}(\cdot)$.

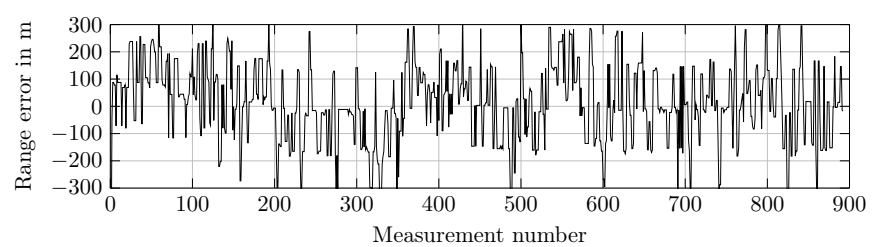

(b) ToF $d_{\mathrm{ToF}, \mathrm{MC}}$ ranging error.

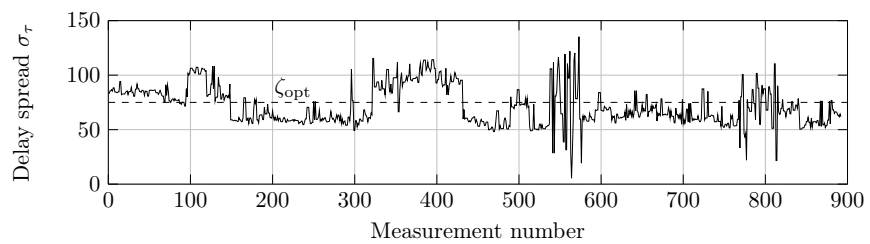

(c) Estimated delay spread $\sigma_{\tau}$ based on the measured CIR.

Fig. 5. PoF/ToF ranging errors and delay spread for the 900 measurements.

ToF $d_{\text {ToF,MC }}$ ranging errors are illustrated in Fig. $5 \mathrm{~b}$. PoF based estimates reveal a higher precision compared to $\mathrm{ToF}$ based ranging, due to the improved temporal resolution thanks to the virtually increased bandwidth, which allows at the same time conserving high receiver sensitivity for long-range communication. The Cumulative Distribution Functions (CDFs) in Fig. 8 show how PoF ranging outperforms ToF based range estimation.

However, estimates show large position dependent biases. For example, in measurements 1 to 100 , a bias is observed corresponding to a multipath reflection $p_{1}$ via the building $b_{2}$, stronger than the direct path obstructed by the edge of building $b_{1}$ (see Fig. 4).

Fig. 6 gives an example of the estimated CIR $h(\tau)$ for biased and bias free measurements, motivating their classification based on the delay spread [9]. This hypothesis is supported by the correlation of biases and delay spread in Fig. 5a and Fig. 5c. Estimates with range bias expose larger

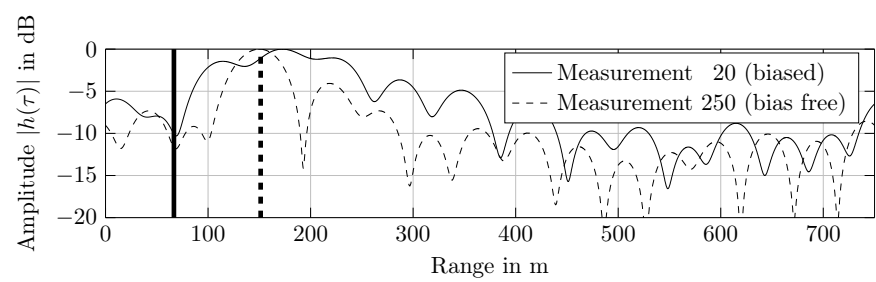

Fig. 6. Comparison of estimated channel impulse responses $h(\tau)$ for a biased and bias free measurement. Vertical lines indicate true range. 


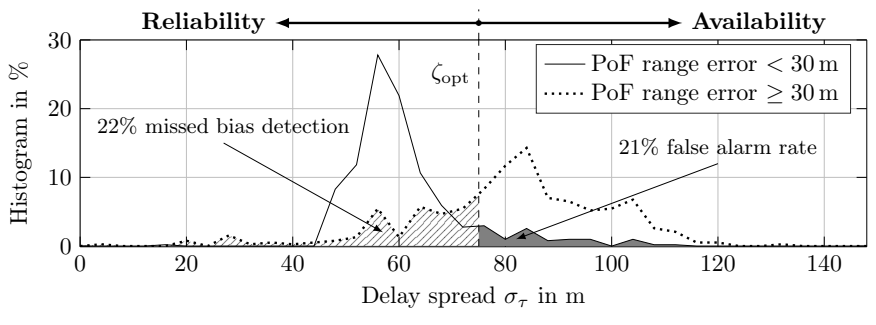

Fig. 7. Delay spread histograms for biased PoF ranging estimates detection.

delay spread than range bias free measurements.

Classification is performed by eliminating $\mathrm{PoF}$ range estimates for which the delay spread exceeds a threshold $\zeta_{\text {opt }}$. These samples are deemed not reliable. In order to determine this threshold, two measurement sets are generated depending on whether PoF errors exceed or not $30 \mathrm{~m}$. Fig. 7 shows the delay spread histograms for these sets of bias free and biased classified measurements. Biased estimates can be detected and removed by considering only estimates for which $\sigma_{\tau}<\zeta_{\text {opt }} \approx 75 \mathrm{~m}$. This corresponds to retaining $62 \%$ of all measurements, a missed bias detection of $22 \%$ and a $21 \%$ false alarm rate. Fig. 5a shows the range estimates obtained by searching for the first path in the CIR and classified as reliable in bold. Other thresholds $\zeta_{\text {opt }}$ as trade-off between ranging measurement reliability and availability are possible as illustrated by the CDFs in Fig. 8 .

PoF ranging in combination with range bias classification clearly outperforms legacy ToF based range estimation as summarized by the CDFs in Fig. 8 .

\section{CONCLUSION}

Multi-channel PoF based ranging achieves $5 \mathrm{~m}$ precision in an AWGN channel. The LPWA ranging field trials presented in this paper reveal how multi-channel ranging improves in $90 \%$ of the cases to range errors smaller than $60 \mathrm{~m}$, compared to ToF based range estimation $(250 \mathrm{~m})$. Coherent combination of narrowband LPWA signals allows reconstructing an estimate of the CIR with a temporal resolution inversely proportional to the virtual bandwidth of the multi-channel scheme. A virtual bandwidth of $3 \mathrm{MHz}$ covers the $868 \mathrm{MHz}$ ISM band with a reasonable number of channels. Hence, the CIR measurement provides precise range estimation and enables the application of methods

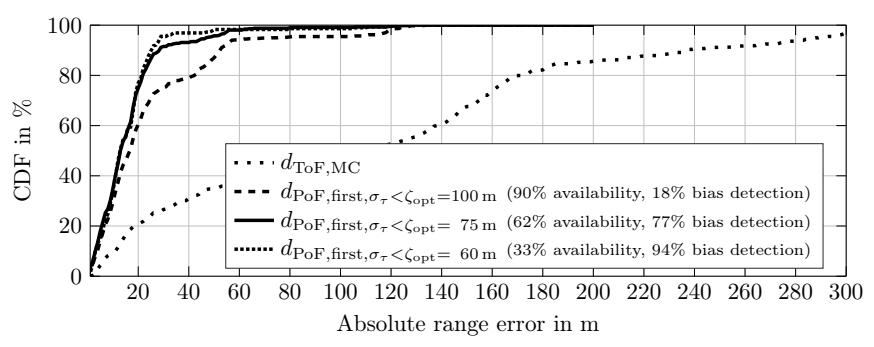

Fig. 8. Ranging errors for ToF and PoF estimation. that derive information on the reliability of range estimates. The virtually increased bandwidth allows resolving multipath components and weaker first paths can be detected, mitigating range biases. Large ranging biases correlate with larger delay spreads. Consequently, biased range estimates can be detected and eliminated. The proposed method, combining first path detection and outlier elimination achieves $30 \mathrm{~m}$ in $90 \%$ of the cases. Providing this reliability information allows, in a location-solving algorithm, selecting the range estimates between a wearable health monitoring device and several base stations that are as accurate as possible. In conclusion, multi-channel phase based ranging outperforms time based techniques at equal coverage. These results will be reinforced by more extensive field trials comprising outdoor-indoor scenarios and kilometer-level inter-node ranges.

Future work includes the application of machine learning algorithms to field trial data so to further improve ranging precision. Learning methods do not require a detailed model, as necessary in the parametric framework, but allow finding the best-suited reliability metrics based on data. Combining multi-channel ranging and learning based reliability metrics will provide $10 \mathrm{~m}$-level accurate geolocation for LPWA networks in challenging multipath scenarios. Thus enabling wearable health monitoring applications with positioning.

\section{ACKNOWLEDGMENT}

This work was supported by the European Union's Horizon 2020 research and innovation programme under 5G-HEART project (grant agreement No 857034).

\section{REFERENCES}

[1] P. H. Lehne et al., "D3.1: Healthcare Vertical Trial Requirements Definition and Execution," 5GHEART deliverable, Tech. Rep., October 2019.

[2] M. Aernouts, B. Bellekens, R. Berkvens, and M. Weyn, "A Comparison of Signal Strength Localization Methods with Sigfox," in 2018 15th Workshop on Positioning, Navigation and Communications (WPNC), Oct 2018, pp. 1-6.

[3] M. Skolnik, Radar Handbook, 2nd ed. McGrawHill, 1990.

[4] N. Podevijn, D. Plets, J. Trogh, L. Martens, P. Suanet, K. Hendrikse, and W. Joseph, "TDoA-Based Outdoor Positioning with Tracking Algorithm in a Public LoRa Network," Wireless Communications and Mobile Computing, 2018.

[5] Semtech, "SX1280/SX1281 Long Range, Low Power, $2.4 \mathrm{GHz}$ Transceiver with Ranging Capability," 2017.

[6] D. Vasisht, S. Kumar, and D. Katabi, "Decimeter-Level Localization with a Single WiFi Access Point," 2016.

[7] M. Pichler, S. Schwarzer, A. Stelzer, and M. Vossiek, "Multi-Channel Distance Measurement With IEEE 802.15.4 (ZigBee) Devices," IEEE Journal of Selected Topics in Signal Processing, vol. 3, no. 5, pp. 845-859, Oct 2009.

[8] A. Povalac and J. Sebesta, "Phase Difference of Arrival Distance Estimation for RFID Tags in Frequency Domain," in 2011 IEEE International Conference on RFID-Technologies and Applications, Sept 2011, pp. 188-193.

[9] S. Marano, W. M. Gifford, H. Wymeersch, and M. Z. Win, "NLOS Identification and Mitigation for Localization based on UWB Experimental Data," IEEE Journal on Selected Areas in Communications, vol. 28, no. 7, pp. 1026-1035, Sep. 2010.

[10] F. Wolf, J.-B. Dore, X. Popon, S. de Rivaz, F. Dehmas, and J. P. Cances, "Coherent Multi-Channel Ranging for Narrowband LPWAN: Simulation and Experimentation Results," in 15th Workshop on Positioning, Navigation and Communications (WPNC), October 2018, pp. 1-6. 